\title{
Azacytidine for Concurrent Acute Monocytic Leukemia and Multiple Myeloma in an Elderly Patient
}

\author{
Unal ATAS ${ }^{1}$, Gokhan TAZEGUL ${ }^{2}$, Orhan Kemal YUCEL ${ }^{1}$, \\ Turgay ULAS ${ }^{3}$, Ozan SALIM ${ }^{1}$, Levent UNDAR ${ }^{1}$

\footnotetext{
${ }^{1}$ Akdeniz University Faculty of Medicine, Department of Hematology, Antalya, TURKEY

${ }^{2}$ Ankara Polatlı Duatepe State Hospital, Department of Internal Medicine, Ankara, TURKEY

${ }^{3}$ Near East University Faculty of Medicine, Department of Hematology, Nicosia, CYPRUS
}

To the editor,

Simultaneous occurrence of MM and AML is a rare entity. ${ }^{1,2}$ Herein, we report an elderly patient with simultaneous MM and AML, successfully treated with azacytidine.

A seventy-one year old male patient was referred with fatigue, immune-paresis and IgG-Lambda monoclonal gammopathy. Physical examination was unremarkable. Complete blood counts were hemoglobin: $13 \mathrm{~g} / \mathrm{dL}(12-16)$, platelets: $175 \times 10^{9} / \mathrm{L}$ (150-450), leukocytes: $3.3 \times 10^{9} / \mathrm{L} \quad(3.91-8.77)$, neutrophils: $1.8 \times 10^{9} / \mathrm{L} \quad(2.06-7.02)$, monocytes: $0.5 \times 10^{9} / \mathrm{L}(0.3-0.8)$. Serum calcium and creatinine levels were normal. Several lytic lesions were detected in cervical vertebrae by magnetic resonance imaging. Bone marrow samples showed CD38, CD138, CD56 and lambda positive, CD19 negative, 10-20\% monoclonal plasma cells. Karyotyping revealed Y chromosome loss. Fluorescent in situ hybridization (FISH) was negative for common, and/ or prognostically significant chromosomal aberrations. Patient was diagnosed with symptomatic MM and bortezomib-cyclophosphamide-dexamethasone (VCD) regimen was started. He was admitted with pneumosepsis and pancytopenia at the end of first cycle. During follow-up, leukocytosis gradually developed within ten days (leukocytes: $35.6 \times 10^{9} / \mathrm{L}$,

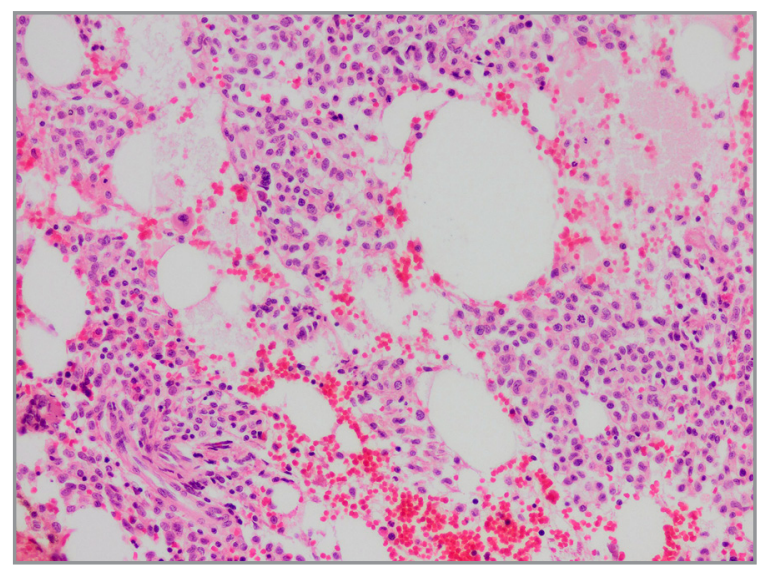

Figure 1. Monoclonal plasma cell infiltration, along with myeloid maturation arrest a lesser monocytic component was detected (H\&E, x200)

neuthrophils: $9.7 \times 10^{9} / \mathrm{L}$ lymphocytes: $3.2 \times 10^{9} / \mathrm{L}$ monocytes: $0.5 \times 10^{9} / \mathrm{L}$ ). Monocytes and monoblasts were markedly increased in blood smear. In a repeat bone marrow aspiration, CD64, CD33, HLADR, CD11b, CD11c positive; CD34, CD117, MPO, CD13, CD14 negative, $86 \%$ monocytic infiltration was observed; concurrent monoclonal plasma cells were undetectable. Karyotyping was 46,XY and both MM and AML-FISH panel were negative. FLT3-ITD PCR was negative, nucleophosmin (NPM1) PCR was positive for type A mutation. 


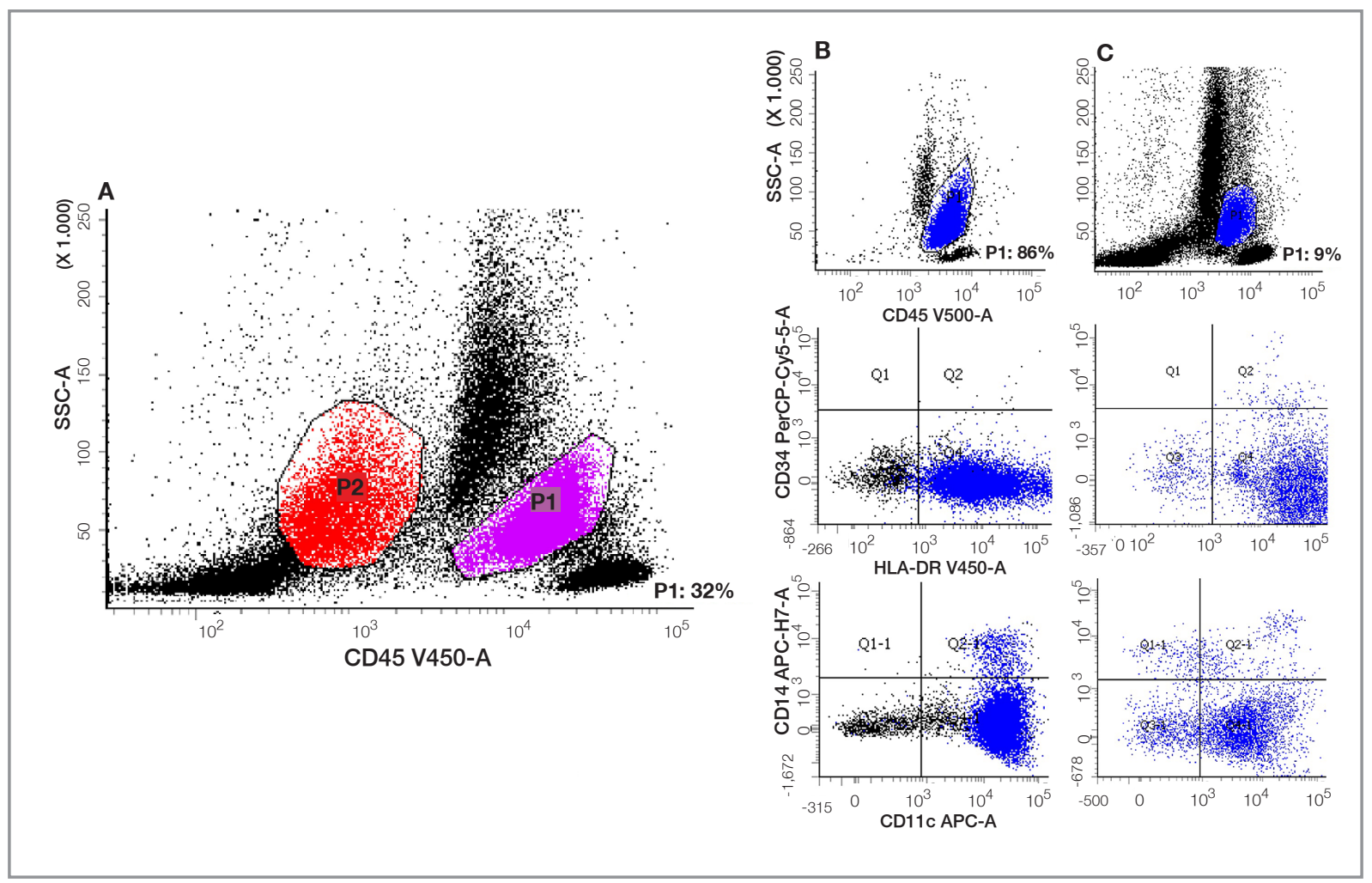

Figure 2. Flow cytometric evaluation of bone marrow at (i) the time of MM diagnosis (A), (ii) the time of $A M L$ diagnosis (B) and (iii) post-treatment (C). Red color shows MM cells. Blue color shows monocytic blastic component. Purple color shows morphologic distribution of monocytic component at the time of MM diagnosis. Each row is the continuation of the scatterplot of same markers at different times of treatment. Column A shows CD45 and sidescatter. For columns B and C, first row shows CD45 and sidescatter, second row shows CD34 and HLA-DR, third row shows CD14 and CD11C stains. Percentages are shown below each scatterplot.

When the first bone marrow samples were reexamined, myeloid maturation arrest and a lesser monocytic component was detected; along with monoclonal plasma cells (Figure 1).

Patient was diagnosed as AML and MM. Azacytidine monotherapy $\left(75 \mathrm{mg} / \mathrm{m}^{2} /\right.$ day, 7 days, 28 -daycycle) was started. Both MM and AML partially responded (3\% clonal plasma cells and $9 \%$ monocytic population) to treatment at the end of 4th cycle with complete hematological recovery (Figures 2, 3).

Patient was clinically stable for 15 cycles. After 15 cycles, he was admitted with pneumosepsis and pancytopenia. Bone marrow samples showed both $22 \%$ monoclonal plasma cells and 35\% monocytes. MM and AML had lost response to therapy, and patient succumbed to septic shock.

It is unclear how AML and MM occur concurrently; several genetic defects were associated with this rare occurance. ${ }^{3}$ Although our patient was diagnosed with AML after the initial cycle of VCD, we classified the patient as concurrent AML and MM, rather than secondary AML to chemotherapy, as we could determine a monocytic component by reviewing previous bone marrow samples. We hypothesize that the proliferation of myeloid clone may have increased by immunosuppression after the initial VCD treatment.

We determined our treatment strategy over AML, since AML would be the determinant of overall survival. Concurrent prognosis is noted to be poor, due to rarity of the disease and no standard treatment. ${ }^{2,3}$ Azacytidine was previously shown to induce apoptosis, upregulate proinflammatory cytokine pathways and cytotoxicity, as well as necrosis in MM cells. ${ }^{4}$ Therefore, azacytidine monotherapy was chosen. Azacytidine was effective on both AML and MM clones for 15 months. This regimen could be a reasonable option for future cases with similar diagnosis. Further studies are warranted in order to improve clinicobiological understanding. 

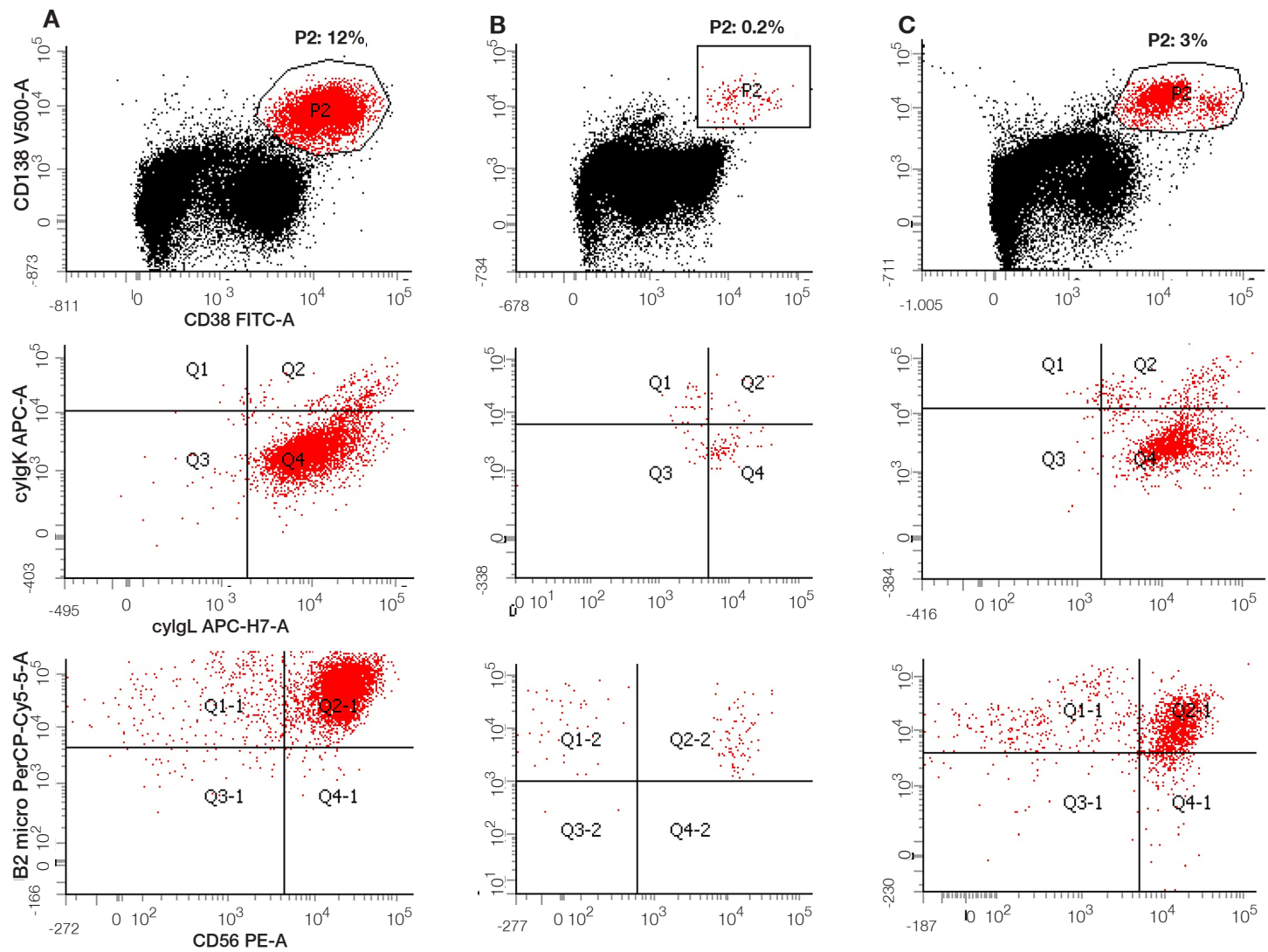

Figure 3. Flow cytometric evaluation of bone marrow at (i) the time of MM diagnosis (A), (ii) the time of AML diagnosis (B) and (iii) post-treatment (C). Each row is the continuation of the scatterplot of same markers at different times of treatment. Red color shows MM cells. First row shows CD38 and CD138, second row shows cylgK and cylgL, third row shows B2 microglobin and CD56 stains. Percentages are shown below each scatterplot.

\section{Acknowledgments:}

We thank Dr. Bahar Akkaya, Professor of Pathology, Akdeniz University School of Medicine; for providing microscopic findings of the bone marrow.

\section{REFERENCES}

1. Oka S, Ono K, Nohgawa M. Successful treatment with azacitidine for the simultaneous occurrence of multiple myeloma and acute myeloid leukemia with concomitant del(5q) and the JAK2 V617F mutation. Ann Hematol 96: 1411-1413, 2017.

2. Murukutla S, Arora S, Bhatt VR, et al. Concurrent Acute Monoblastic Leukemia and Multiple Myeloma in a 66-Year-Old Chemotherapy-Naive Woman. World J Oncol 5: 68-71, 2014.

3. Lu-qun W, Hao L, Xiang-xin L, et al. A case of simultaneous occurrence of acute myeloid leukemia and multiple myeloma. BMC Cancer 15: 724, 2015.
4. Tian E, Tang H, Xu R, et al. Azacytidine induces necrosis of multiple myeloma cells through oxidative stress. Proteome Sci 11: 24, 2013.

\section{Correspondence:}

Dr. Unal ATAS

Akdeniz Üniversitesi Tip Fakultesi

Hematoloji Anabilim Dali Konyaalti, ANTALYA / TURKEY

Tel: $\quad(+90-555) 6374362$

Fax: (+90-242) 2496903

e-mail:vrlunalatas@gmail.com

\section{ORCIDs:}

Unal Atas:

$0000-0001-5897-6514$

Gokhan Tazegul:

Orhan Kemal Yucel:

0000-0002-0737-9450

Turgay Ulas:

$0000-0002-0455-1382$

Ozan Salim:

$0000-0001-9332-663 X$

0000-0001-6687-0189

Levent Undar:
0000-0002-9853-5075 